\title{
Jahrestagung der Deutschen Gesellschaft für Phlebologie
}

\section{Aachen 2021 - Synergie und Vielfalt (www.phlebologie-2021.de)}

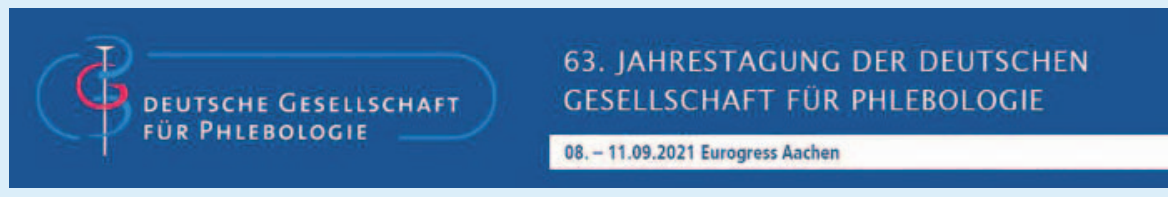

Die 63. Jahrestagung unserer Gesellschaft findet in diesem Jahr vom 8.-11. September im Eurogress in Aachen unter dem Tagungsmotto „Aachen 2021 - Synergie und Vielfalt" statt.

Schwerpunkte der Tagung werden die Präsentation des wissenschaftlichen Fortschritts und aktuelle Innovationen, die Versorgungssituation in der Praxis und die Fortbildung sowohl für den niedergelassenen Arzt als auch für den jungen Phlebologen sein. Wir freuen uns, Ihnen auch in diesem Jahr wieder einen internationalen
Strang, der simultan übersetzt wird, anbieten zu können.

Das bewährte Kurssystem (Crashkurs, Update, zahlreiche Hands-on-Kurse) behalten wir bei - nutzen Sie hier die Gelegenheit: praxisrelevante Therapieformen von erfahrenen Phlebologen gezeigt und von Ihnen als Teilnehmer geübt.

Speziell für junge Phlebologen haben wir in diesem Jahr einen Weiterbildungspfad konzipiert (siehe Kasten) - hier besteht die Möglichkeit, sich aktiv theoretisch und praktisch phlebologisch fortzubilden. Die
Anmeldung auf der Kongress-Homepage https://phlebologie-2021.de/ bietet Ihnen hier attraktive Konditionen.

Darüber hinaus werden neben den klassischen phlebologischen Themen des interdisziplinär besetzten Fachbereichs wie Kompression, Sklerosierung, operative und endovenöse Therapie der Varikose auch die Ästhetik in der Phlebologie, Wundversorgung, Antikoagulation und Berufspolitik ausführlich diskutiert werden. Ein weiterer wesentlicher Schwerpunkt wird auf das tiefe Venensystem gerichtet sein: interventionelle und operative Therapie bei akuter Thrombose und chronischer Obstruktion, Kompressionssyndrome und Pelvic-Congestion-Syndrom.

Wir freuen uns, Ihnen darüber hinaus am Samstag, 11. September 2021, ganztägig 
einen DEGUM-Aufbaukurs „Haut, Subkutis und Lymphknoten“ anbieten zu können.

Anmeldungen zu den Kursen sind schon jetzt unter https://phlebologie-2021.de/ anmeldung/ möglich.

Der intensive Dialog der Teilnehmer aus allen Fachbereichen und Berufsgruppen der Phlebologie und ihrer Nachbardisziplinen ist uns ein großes Anliegen. Nur die enge Vernetzung der beteiligten Fachbereiche ermöglicht eine optimale Patientenversorgung. Gemeinsame Sitzungen mit den befreundeten Fachgesellschaften sind geplant.

Auch gibt es bereits die Möglichkeit zur Einreichung Ihrer Abstracts unter: https://phlebologie-2021.de/call-forabstracts-2021/.

Auch in Aachen haben wir das große Glück, mit einer großen Zahl an großen und kleineren Vortragssälen planen zu können, sodass Abstandsregelungen, die u. U. noch gelten, eingehalten werden können.

Wir freuen uns, wenn viele von Ihnen den Weg nach Aachen finden - wissenschaftlicher Austausch nach der langen Durststrecke tut uns allen sicher gut.

Dr. med. Knut Rass

Dr. med. Houman Jalaie
WEITERBILDUNGSPFAD

PHLEBOLOGIE FÜR JUNGE

PHLEBOLOGEN

- Crashkurs Phlebologie

- Weiterbildungsforum

- Workshop Sklerotherapie

- Workshop Operative Therapie der Varikose

- Workshop Endovenöse Obliterationsverfahren

- Workshop Venous Stenting

- Workshop Kompression

WISSENSCHAFTSPREISE, DIE IM RAHMEN DER JAHRESTAGUNG VERLIEHEN WERDEN

- 2 Rednerpreise à 500 Euro für die besten Präsentationen im Rahmen der Jahrestagung

- Bauerfeind-Doktorandenpreis für junge Mediziner mit wissenschaftlichem Interesse auf den Gebieten Phlebologie, Lymphologie und verwandten Disziplinen

- Kreussler Young Phlebologists' Travel Award zur Förderung des wissenschaftlichen Nachwuchses

- medi Reise- und Teilnahmestipendien für Arzt-Berufsstarter

- Sigvaris-Förderpreis Phlebologie für junge Wissenschaftler und
Mediziner mit Interesse an wissenschaftlicher Arbeit

- Juzo-Forschungspreis Phlebologie zur Förderung der Wissenschaft in der interdisziplinären Kompressionstherapie für Mediziner mit wissenschaftlichem Interesse auf den Gebieten Phlebologie, Lymphologie, Dermatologie und verwandten Disziplinen

- Zoltan-Varady-Vortragspreis für den besten Vortrag im Bereich der Minichirurgie oder Mikrochirurgie

Die Preise bieten Nachwuchswissenschaftlern z. B. die Möglichkeit einer finanziellen Unterstützung bei einem wissenschaftlichen Projekt. Bitte weisen Sie Ihre jungen Kollegen und Kolleginnen auf diese interessante Fördermöglichkeit hin.

Ausführliche Beschreibungen der Preise finden Sie auf: https://phlebologie2021.de/wissenschaftspreise/.

\section{IMPRESSUM}

Verantwortlich für Mitteilungen der DGP:

Dr. med. Erika Mendoza 